\title{
On a Search for Hidden Photon CDM by a Multicathode Counter
}

\author{
A. V. Kopylov, I. V. Orekhov, and V. V. Petukhov \\ Institute for Nuclear Research of Russian Academy of Sciences, Prospect of 60th Anniversary of October Revolution 7A, \\ Moscow 117312, Russia \\ Correspondence should be addressed to A. V. Kopylov; beril@inr.ru
}

Received 23 March 2016; Accepted 23 May 2016

Academic Editor: Hiroyasu Ejiri

Copyright @ 2016 A. V. Kopylov et al. This is an open access article distributed under the Creative Commons Attribution License, which permits unrestricted use, distribution, and reproduction in any medium, provided the original work is properly cited. The publication of this article was funded by SCOAP ${ }^{3}$.

\begin{abstract}
We report a new technique of a multicathode counter (MCC) developed to search for hidden photon (HP) cold dark matter (CDM) with a mass from 5 to $500 \mathrm{eV}$. The method is suggested in the assumption that HP-photon mixing causes emission of single electrons from a metal cathode if the mass of hidden photon $m_{\gamma^{\prime}}$ is greater than a work function of the metal $\varphi_{W}$. The measured effect from HP should be dependent on $\varphi_{W}$ and on the structure of electronic shells of the metal used as a cathode. Potentially this can be used for a verification of the results obtained. Some preliminary results for the upper limit for mixing parameter $\chi$ have been obtained for $\mathrm{HP}$ with a mass from $5 \mathrm{eV}$ to $10 \mathrm{keV}$ as a pure illustration of the potential of this technique. The efforts are continued to refine the procedure of data treatment and to improve the work of MCC. A new detector with a more developed design is under construction.
\end{abstract}

\section{Introduction}

The present data on the structure formation in the Universe indicate that most dark matter (DM) is "cold"; that is, it should be nonrelativistic. Neutrino in a hot dark matter concept can be envisaged only in combination with cold dark matter (CDM). Now the most attractive DM candidates appear to be Weakly Interactive Massive Particles (WIMP). Great progress in this field of research is outlined in [1]. However, there are other alternatives, among them axion and axion-like particles (ALP) which is probably a next most promising field. The efforts towards discovering axion are described in detail in [2]. Another interesting opportunity is a hidden photon which is a light extra $U(1)$ gauge boson. As it was suggested in $[3,4]$ hidden photons (HP) may be observed in experiment through a kinetic mixing term $(\chi / 2) F_{\mu \nu} X^{\mu \nu}$ with the ordinary photons, where $\chi$ is a parameter quantifying the kinetic mixing. Here $F_{\mu \nu}$ is the field stress of the ordinary electromagnetic field $A^{\mu}$ and $X^{\mu \nu}$ is the field stress of the HP field $X^{\mu}$.

Recently the $\mathrm{eV}$ mass range of $\mathrm{HP}$ was investigated with a dish antenna [5], a novel method proposed in [6]. The idea is to detect electromagnetic wave which is emitted by the oscillation of electrons of the antenna's surface under tiny ordinary electromagnetic field $A^{\mu}$ induced by HP. A dark matter solution for HP with a mass $m_{\gamma^{\prime}}$ reads [6]

$$
\left.\left(\begin{array}{c}
\mathbf{A} \\
\mathbf{X}
\end{array}\right)\right|_{\mathrm{DM}}=\mathbf{X}_{\mathrm{DM}}\left(\begin{array}{c}
-\chi \\
1
\end{array}\right) \exp (-i \omega t)
$$

that is, it has a spatially constant mode $k=0$, oscillating with frequency $\omega=m_{\gamma^{\prime}}$. This method works well only if the reflectance of antenna is high which is observed for $\omega<$ $5 \mathrm{eV}$. In the work [5] using an optical mirror and a photoncounting PMT at the point of convergence of the photons emitted from mirror, the upper limit of $6 \times 10^{-12}$ was obtained for a mixing parameter $\chi$ for the hidden photon mass $m_{\gamma^{\prime}}=$ $3.1 \pm 1.2 \mathrm{eV}$. This work was a first measurement of $\chi$ within this range of $m_{\gamma^{\prime}}$ using a dish antenna. Our work was the search for hidden photons for the upper range of $m_{\gamma^{\prime}}$ using a gaseous proportional counter as a detector of electrons emitted from a metal cathode by hidden photons. This constitutes the novelty of our method.

\section{A Method and Experimental Apparatus}

The principal difference of our approach from [6] is that here we focus on shorter wavelengths, that is, higher masses of 


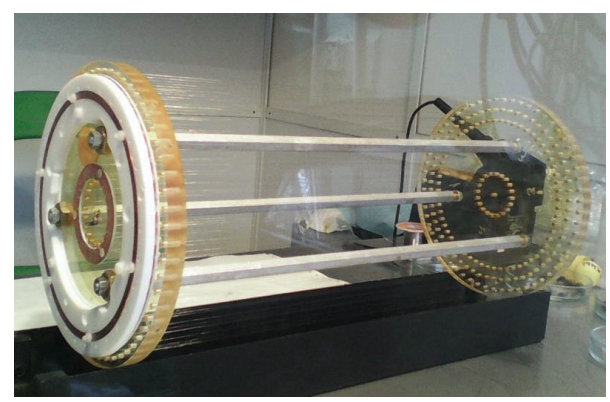

FIGURE 1: The central part of the counter.

HPs for which the reflectance of antenna is low. We make an assumption that in this case a HP-photon conversion will cause emission of single electrons from the surface of antenna similar to what one observes when metal is exposed to UV radiation. To register this conversion the detector should be highly sensitive to single electrons emitted from metal. Here we would like to draw attention to the fact that the detector in this case is sensitive exclusively to HP-photon conversion, not to photon-HP conversion. For the latter this method does not work. We assume here that probability for the electron to be emitted after a hidden photon gets converted into ordinary electric field in a metal cathode would be equal to quantum efficiency $\eta$ for a given metal to emit electron after absorption of a real UV photon of energy $\omega=m_{\gamma^{\prime}}$. The details of how electrons are created after photons get absorbed by a metal photocathode are described in many papers; see, for example, [7]. The obvious difference between these two processes is that UV photons are strongly absorbed by the metal, while HPs move freely through the metal, but this leads only to some underestimation of the effect from HP-photon conversion. We did not take this into account. Neither did we take into account the effects of surface roughness of the cathode. We consider this to be small corrections and leave it for our further study. To make a practical implementation of this idea a special technique of a multicathode counter (MCC) has been developed and some very preliminary data as a pure illustration of the potential of this technique in the search for hidden photons has been obtained.

To count electrons emitted from a metal cathode we used a gaseous proportional counter filled by argon-methane (10\%) mixture at $0.2 \mathrm{MPa}$. To detect single electrons the counter should have high $\left(\geq 10^{5}\right)$ coefficient of gas amplification. The general view of the counter is presented in Figure 1 and the electronic scheme in Figure 2. Present design of MCC first described in [8] is a further development of the work with the aim of making an apparatus to register neutrinonucleus coherent scattering $[9,10]$. The cathode of the counter is $194 \mathrm{~mm}$ in diameter and $400 \mathrm{~mm}$ in length. It has relatively large $\left(\approx 0.2 \mathrm{~m}^{2}\right)$ surface which acts in this experiment as "antenna" for HP but instead of reflecting electromagnetic waves it emits single electrons. The counter has a central anode wire of $20 \mu \mathrm{m}$ and 4 cathodes; 3 of them are composed of an array of $50 \mu \mathrm{m}$ nichrome wires tensed with a pitch of a few $\mathrm{mm}$ around anode one after another, and a fourth one, more distant from anode, is a cathode made of copper.
The apparatus is counting electrons emitted from the walls of a cathode at short wavelengths $\omega=m_{\gamma^{\prime}} \approx 5-$ $500 \mathrm{eV}$. The diameter of the first cathode $D_{1}$ is $40 \mathrm{~mm}$ to ensure high $\left(\geq 10^{5}\right)$ coefficient of gas amplification in the central section of the counter. Three different configurations of the same counter are used to measure the count rate of single electrons. In the first configuration electrons, emitted from copper, drift freely to the central section with high gas amplification. The highest negative potential is applied in this configuration to the copper cathode. The rate $R_{1}$ measured in this configuration is as follows:

$$
R_{1}=R_{\mathrm{Cu}}+R_{\mathrm{sp}} .
$$

Here $R_{\mathrm{Cu}}$ is the count rate from single electrons emitted from copper and $R_{\mathrm{sp}}$ is the rate from spurious pulses generated in the volume limited by a diameter $194 \mathrm{~mm}$. What is the origin of spurious pulses? This question will be the subject of our further study. Here we assume that they are produced by "hot" spots on the surface of metal and are the effect of microstructure of a metal. The microprotrusions (spearheads) on the surface of wires may generate single electrons in strong electric fields. The spots with impurities of other metals, especially alkaline, may generate emission of single electrons and so forth. Only the future work can show how successful can this approach be.

In the second configuration the highest negative potential is applied to the third cathode $D_{3}=180 \mathrm{~mm}$. In this configuration the count rate is as follows:

$$
R_{2}=0.11 \cdot R_{\mathrm{Cu}}+R_{\mathrm{sp}}
$$

The factor 0.11 was obtained by calibration of the counter in 1st and 2 nd configurations by UV source of the same intensity. Here the key point is that in 1st and 2nd configurations the counter has approximately the same geometry and the same wires. So if "hot" spots are on the surface of wires, the difference of the count rates in 1st and 2nd configurations will contain only $89 \%$ of the count rate of single electrons emitted from copper cathode. Because the geometry of the counter in 1st configuration is very similar to its geometry in 2 nd configuration the same reasoning is valid for spurious pulses generated by a leakage current through dielectric used in the construction of the counter.

In the third configuration the highest negative potential is applied to a second cathode $D_{2}=140 \mathrm{~mm}$. The rate $R_{3}$ measured in this configuration is determined by spurious pulses generated within a volume limited by smaller diameter $140 \mathrm{~mm}$ with different number of wires and different isolators, so

$$
R_{3}=r_{\mathrm{sp}}
$$

In experiment the rate $R_{3}$ turned out to be approximately 3 times smaller than the rates $R_{1}$ and $R_{2}$ (which are very close) not in contradiction with our explanation of the origin of the spurious pulses. All this explains why as a measure of the effect from HPs we use the count rate

$$
R_{\mathrm{MCC}}=\frac{\left(R_{1}-R_{2}\right)}{0.89}=R_{\mathrm{Cu}} \text {. }
$$




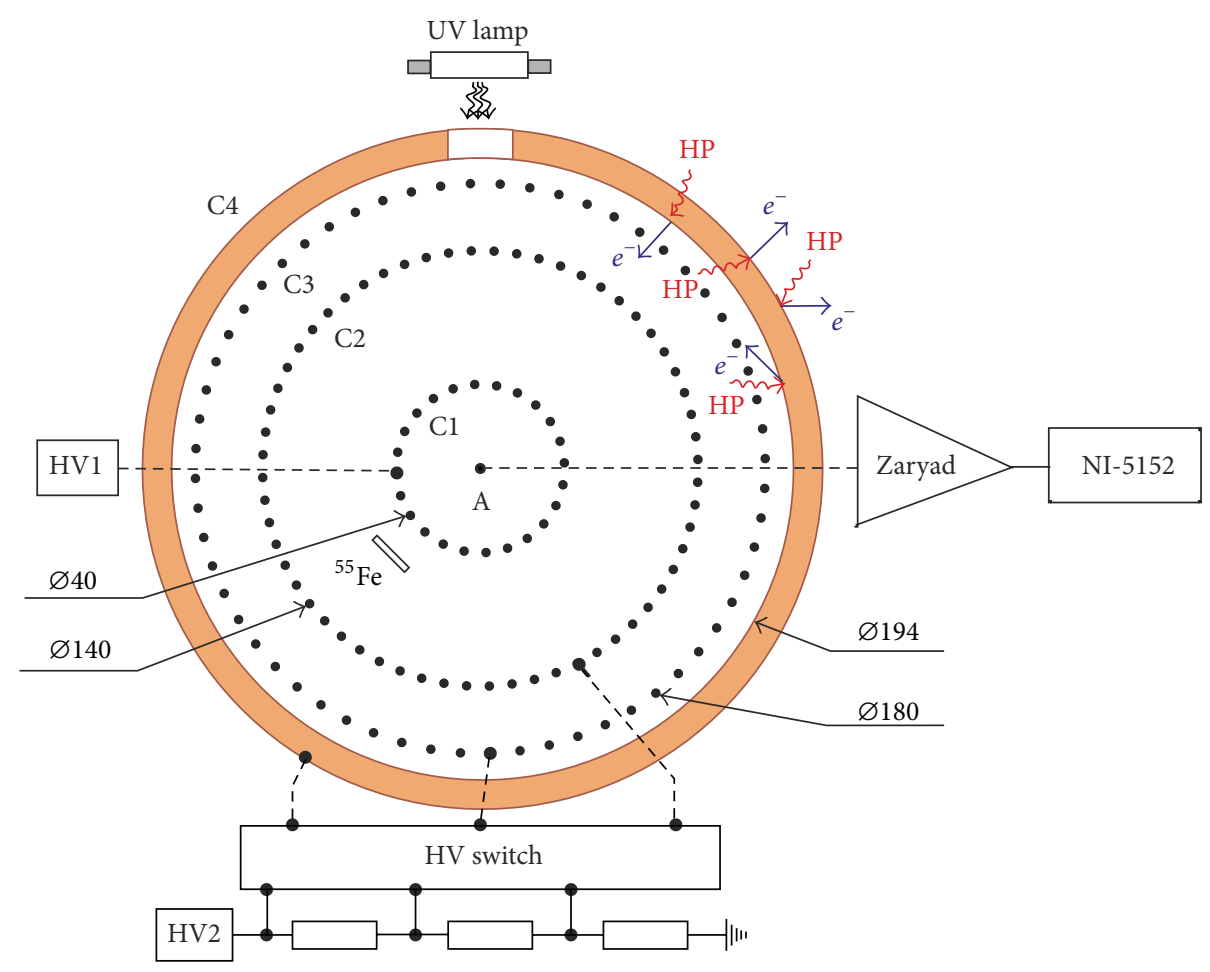

Figure 2: A simplified electronic scheme of a multicathode counter (MCC). A: anode, C1-C4: cathodes, and "Zaryad": charge sensitive preamplifier.

The measurements were performed in each configuration by switching the counter consecutively in three different configurations, by calibration of the counter, and by measuring the rates $R_{1}, R_{2}$, and $R_{3}$. Then from a number of measured points the average rates $\bar{R}_{1}, \bar{R}_{2}$ were found and from here the average rate $\bar{R}_{\mathrm{MCC}}$ is found. Then from the scattering of the experimental points the uncertainties were calculated for each rate and, finally, for $R_{\mathrm{MCC}}$. The count rate $R_{3}$ has been used to monitor the counting process to exclude some possible interference by external sources of electromagnetic disturbances.

\section{Energy Calibration and Analysis}

The calibration of the counter has been conducted by ${ }^{55} \mathrm{Fe}$ source and by UV light of the mercury lamp. The calibration by ${ }^{55} \mathrm{Fe}$ source was used to determine at what high voltages, HV1 and HV2, the counter was working in a mode of limited proportionality with high $\left(>10^{5}\right)$ gas amplification. Here we followed the standard technique described in many papers, for example, in [11]. It was also described in our earlier papers $[9,10]$. High voltage at first cathode was $2060 \mathrm{~V}$ and the ones from the voltage divider have been used for all three configurations such as to ensure the amplitude of the pulse corresponding to peak $5.9 \mathrm{keV}$ from $\mathrm{K}$-line of ${ }^{55} \mathrm{Mn}$, which is eradiated as a result of $\mathrm{K}$-electron capture by ${ }^{55} \mathrm{Fe}$, on the output of charge sensitive preamplifier to be at the level $1400 \mathrm{mV}$ which corresponds to a gas amplification $A \approx 10^{5}$. Figure 3 shows the calibration spectrum.

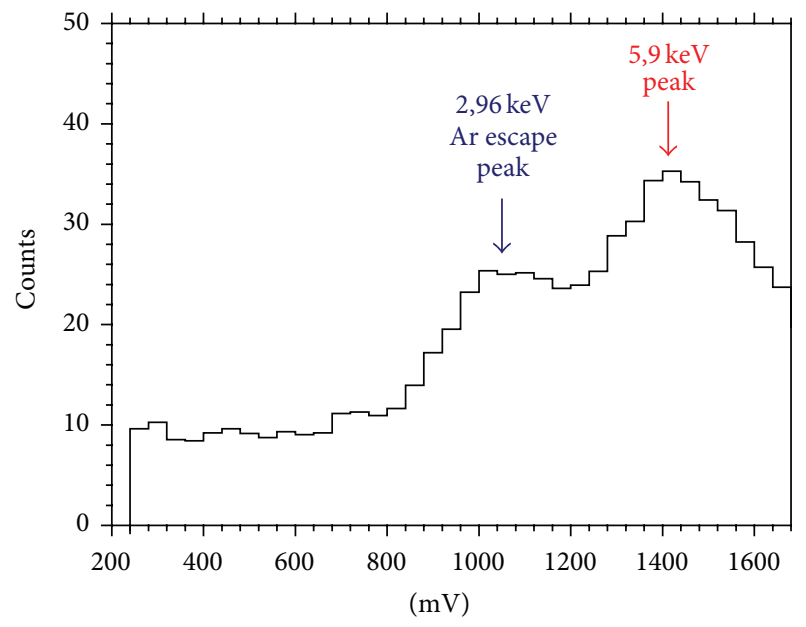

FIGURE 3: The calibration spectrum from ${ }^{55} \mathrm{Fe}$ source introduced directly in a working volume of the counter facing first cathode.

The amplitude of the escape peak of argon at the energy $2.96 \mathrm{keV}(5.9 \mathrm{keV}$ minus $2.94 \mathrm{keV}$ of $\mathrm{K}$ X-ray of argon escaping the detection region) was shifted from the due position in case of a linear response at about $700 \mathrm{mV}$ to the one at approximately $1000 \mathrm{mV}$. This nonlinearity in the spectrum indicates that the counter was working in the regime of limited proportionality. From the approximation done through three points, zero and two peaks, $5.9 \mathrm{keV}$ at $1400 \mathrm{mV}$ and $2.96 \mathrm{keV}$ at $1000 \mathrm{mV}$, one can find that at 


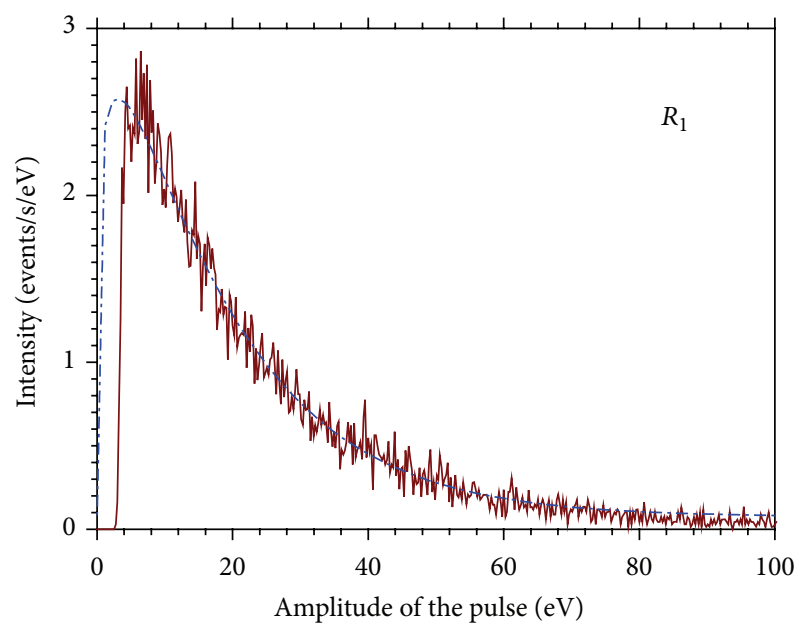

(a)

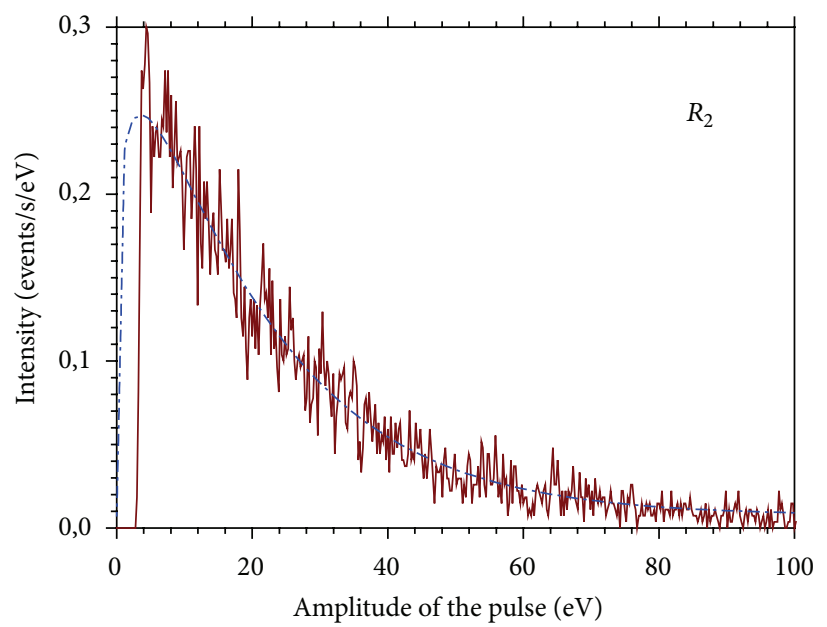

(b)

FIgURE 4: The single electron spectra obtained in measurements in 1st $\left(R_{1}\right)$ and 2 nd $\left(R_{2}\right)$ configurations at the same flux of UV photons. The dashed curves indicate the Polya distribution.

energies less than $100 \mathrm{eV}$ a gas amplification $A \approx 1.8 \cdot 10^{5}$ and the conversion factor is $\approx 2.3 \mathrm{eV} / \mathrm{mV}$. It takes approximately $27 \mathrm{eV}$ to create one electron-ion pair in argon. This means that single electron pulses should be observed in the region below $50 \mathrm{mV}$. The calibration by UV photons demonstrates that this is really so. The ${ }^{55} \mathrm{Fe}$ source has been removed and the internal walls of the counter were irradiated by UV light from a mercury lamp placed outside through a window made of melted silica. The physics and techniques of single electron counting were described in detail in many devoted articles; see, for example, [12-14]. Figure 4 shows the single electron spectra obtained in measurements in 1st and 2nd configurations.

Comparing the count rates $R_{1}$ and $R_{2}$ presented in Figure 4 one can see that in the 2nd configuration the count rate of single electrons was about 0.11 of the rate measured in the first one. It proves that in the 2 nd configuration the electrons emitted from the walls of the counter were really rejected back by the 3rd cathode. This means that the counter in the 2nd configuration can be used for measurement of the background count rate. To compare the gains in different configurations we used the same procedure as the one described in [15]; the inverse indexes of exponents were used as a measure of the gain of the counter. For the pulses above a threshold $7 \mathrm{eV}$ the deviation from exponential distribution was small and could be neglected. The inverse indexes of exponent for all three configurations were in the range $19.3 \pm$ $1.2 \mathrm{eV}$. The counting efficiency for the interval from $7 \mathrm{eV}$ till $70 \mathrm{eV}$ was found to be $76 \pm 5 \%$. This number has been obtained by using Polya distribution [16]:

$$
P(A)=\left(\frac{A(1+\theta)}{\bar{A}}\right)^{\theta} \exp \left(\frac{-A(1+\theta)}{\bar{A}}\right)
$$

where $A$ is gas amplification and $\theta$ is parameter which depends on a working gas and electric field configuration. From the approximation of the measured spectrum by Polya curve it was found that $\theta \approx 0.16$ which is in a reasonable agreement with the expected one for our working gas and electric field configuration. The counting efficiency has been corrected upon the results of calibrations performed in each of three configurations. The possibility to increase the counting efficiency is a subject of our further study. To have a further progress we need to decrease the threshold or to increase the gas amplification.

In the measurements the shapes of the pulses on the output of a charge sensitive preamplifier are recorded by 8bit digitizer. In our previous paper [9] the shapes of "true" and "wrong" pulses are presented. The "true" pulses have typically a relatively short front edge (a few microseconds) corresponding to the drift of positive ions to cathode and long (hundreds of microseconds) tail corresponding to the time of the baseline restoration of the charge sensitive preamplifier. The "wrong" pulses usually have a wrong (too fast or too slow or irregular) front edge or nonexponential tail. In the analyses of the data only pulses with a baseline within $\pm 2 \mathrm{mV}$ were taken with a proper evaluation of the resulted live time. Figures 6 and 7 show the distribution of the events on the diagram "duration of front edge-parameter $\beta$ " for the UV lamp and for real measurements. The parameter $\beta$ is proportional to first derivative of the baseline approximated by a straight line in the interval $50 \mu$ s before the front edge. We used it as a measure of the quality of baseline in the prehistory of the event which has been used in a procedure of automatic screening of all data. The events with $\beta$ beyond the allowed range of $0 \pm 0.1$ were considered as "not having a reliable determined amplitude" events and were discarded from analyses. The statistic collected during a day or even a few days for each configuration was rather high so we could afford to do this discrimination without substantial loss of information. The region of interest (ROI) box contains "true" pulses with amplitude in the interval $[7-70] \mathrm{eV}$ with a front edge in the interval $[2-25 \mu \mathrm{s}]$ and a parameter $\beta$ in the interval $0.0 \pm 0.1$. The pulses beyond this region were 


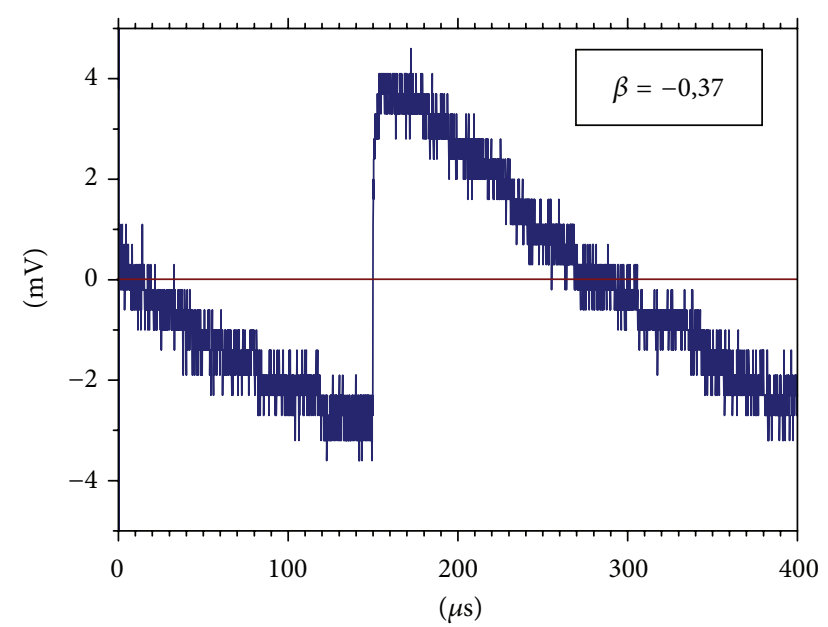

FIGURE 5: One example of the pulse with "bad" prehistory.

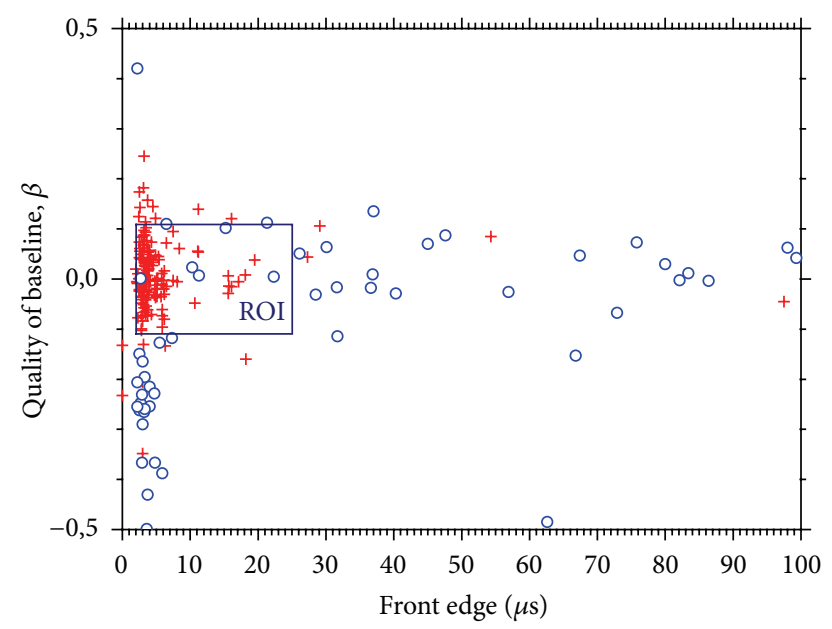

FIGURE 6: The distribution of the events for UV lamp.

rejected as "noisy" pulses. One of the typical "noise" pulses is presented in Figure 5.

One can see that a ROI region contains $95 \pm 5 \%$ of all pulses (crosses) from UV lamp. By inspecting directly a small sample of the real pulses we found that inside of ROI region one can see only about $10 \%$ of the pulses with the "wrong" shape (circles).

To reduce the background from external $\gamma$-radiation the counter has been placed in a cabinet with $30 \mathrm{~cm}$ iron shield. It resulted in decrease of the count rates of single electron events by a factor of 2 (detector on a porch of a cabinet versus detector inside a cabinet) while the flux of gamma rays in the region around $200 \mathrm{keV}$ has been attenuated in these layouts by a factor of 50. From here one gets a simple estimate of the background: inside a cabinet the $\gamma$-radiation cannot be a major source of the single electron events; its contribution is not more than a few percent. The main source is spurious pulses similar to what one observes with PMT; this was also a limiting factor in experiment with a dish antenna [5]. To reduce this dark current we should make further

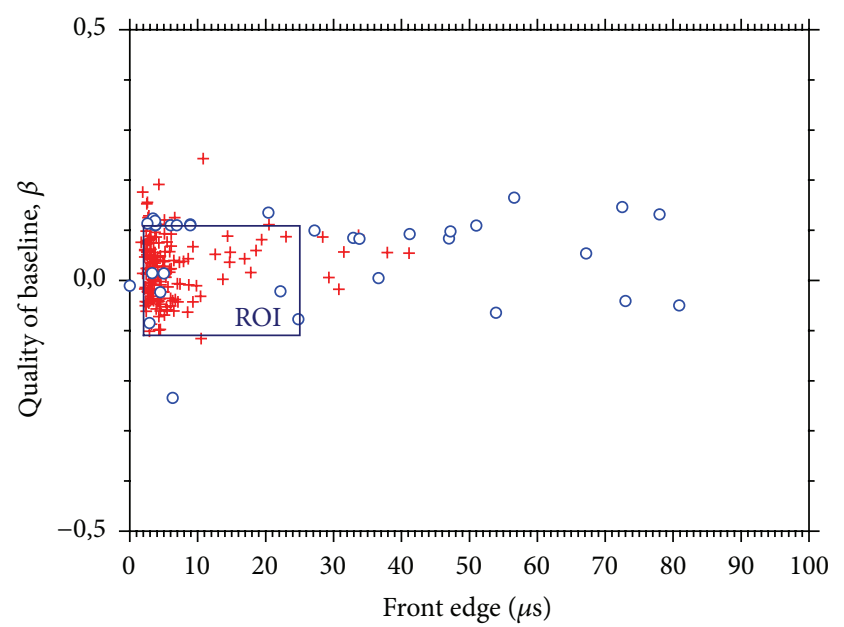

FIgURE 7: The distribution of the real events.

improvements in the construction of the detector. This is our task for future.

The data were collected frame by frame. Each frame contained $2 \mathrm{M}$ points, each point $100 \mathrm{~ns}$. After collection of the data they were stored on a disk and then the collection resumed. The analysis of the collected data was performed offline. The frames with the signs of excessive noisiness were removed from analysis.

\section{Sensitivity of the Method}

Here we follow the same ideology as developed in [6] for a dish antenna with one principal difference: instead of detecting electromagnetic waves we look for single electrons emitted from the surface of antenna. That is, why we use in our experiment not antenna but gaseous proportional counter (see Figure 1). We assume that similar to the emission of single electrons from metal by UV light or by X-rays the probability for the electron to be emitted when HP of the mass $m_{\gamma^{\prime}}$ gets converted into an ordinary electric field in metal is equal to the quantum efficiency $\eta$ for the photon's energy $\omega=m_{\gamma^{\prime}}$. According to [6] if DM is totally made up of hidden photons, the power collected by antenna is

$$
P=2 \alpha^{2} \chi^{2} \rho_{\mathrm{CDM}} A_{\text {dish }}
$$

where $\alpha^{2}=\cos ^{2} \theta, \theta$ is the angle between the HP field, when it points in the same direction everywhere, and the plane of antenna, $\alpha^{2}=2 / 3$ if HPs have random orientation, $\chi$ is the dimensionless parameter quantifying the kinetic mixing, $\rho_{\mathrm{CDM}} \approx 0.3 \mathrm{GeV} / \mathrm{cm}^{3}$ is the energy density of CDM which is taken here to be equal to the energy density of HPs, and $A_{\text {dish }}$ is the antenna's surface. In our case of gaseous proportional counter $P=R_{\mathrm{MCC}} m_{\gamma^{\prime}} / \eta$ and this expression will be as follows:

$$
R_{\mathrm{MCC}} m_{\gamma^{\prime}}=2 \eta \alpha^{2} \chi^{2} \rho_{\mathrm{CDM}} A_{\mathrm{MCC}}
$$


Here $A_{\mathrm{MCC}}$ is the surface of the metal cathode of our counter. From here one can easily obtain

$$
\begin{aligned}
\chi_{\text {sens }}=2.9 & \times 10^{-12}\left(\frac{R_{\mathrm{MCC}}}{\eta 1 \mathrm{~Hz}}\right)^{1 / 2}\left(\frac{m_{\gamma^{\prime}}}{1 \mathrm{eV}}\right)^{1 / 2} \\
& \cdot\left(\frac{0.3 \mathrm{GeV} / \mathrm{cm}^{3}}{\rho_{\mathrm{CDM}}}\right)^{1 / 2}\left(\frac{1 \mathrm{~m}^{2}}{A_{\mathrm{MCC}}}\right)^{1 / 2} \\
& \cdot\left(\frac{\sqrt{2 / 3}}{\alpha}\right) .
\end{aligned}
$$

\section{First Data Obtained}

The detector was placed at the ground floor of a building in Troitsk, Moscow region, in a specially constructed cabinet with $30 \mathrm{~cm}$ iron shield. All count rates were in a few $\mathrm{Hz}$ range. The average value of $R_{\text {MCC }}$ calculated for "quiet" interval during 28 days of measurements was found to be $\bar{R}_{\mathrm{MCC}}=$ $-0.06 \pm 0.36 \mathrm{~Hz}$. The uncertainty has been found from the real scattering of the experimental points. So if we take the normal distribution for uncertainties, then we obtain that, at $95 \%$ confidence level, $\bar{R}_{\mathrm{MCC}}<0.66 \mathrm{~Hz}$. The quantum efficiency $\eta$ was taken from [17] for masses of HPs $m_{\gamma^{\prime}}<$ $11.6 \mathrm{eV}$ (magenta), from [18] for $10 \mathrm{eV}<m_{\gamma^{\prime}}<60 \mathrm{eV}$ (red), from [19] for $20 \mathrm{eV}<m_{\gamma^{\prime}}<10 \mathrm{keV}$ (green), and from [20] for $50 \mathrm{eV}<m_{\gamma^{\prime}}<10 \mathrm{keV}$ (blue). From expression (9) we obtain an upper limit for a mixing constant $\chi$. The values of a mixing constant $\chi$ allowed by this experiment are below the curve presented in Figure 8 . The systematic uncertainty is mainly determined by the uncertainty in quantum efficiency which is taken to be about $30 \%$ following the estimates done in [17]. To decrease this limit one should construct a detector with lower count rate of spurious pulses. The difference in the curves presented in Figure 8 is explained by different purity of copper used in measurements. The data from $[17,20]$ were obtained for atomically clean copper samples prepared by evaporation of copper in high vacuum while routinely cleaned (by solvents) copper samples were used in $[18,19]$. For example, in the paper [21], it was shown that cleaning of the surface of the copper cathode by ionized controlled etching (ICE) can increase the quantum efficiency by an order of magnitude. For atomically clean copper one can see the effect of electronic shells while for routinely cleaned copper the spectra are rather smudged. In our detector for cathode we used routinely cleaned (by solvents) copper. It would be expedient to use a quantum efficiency measured for the specific sample used for a cathode in our detector and we are planning this work for the future. There is a strong dependence of the effect not only on the work function but also on the structure of the electronic shells of the metal used for a cathode. Potentially this can be used for verification that the obtained result is really from HPs. For this we should make measurements using cathodes made of different metals.

The numbers presented in Figure 8 are first very preliminary results for hidden photons with a mass from 5 to $500 \mathrm{eV}$. One can see that the region of highest sensitivity for our method is from 10 to $30 \mathrm{eV}$, that is, approximately

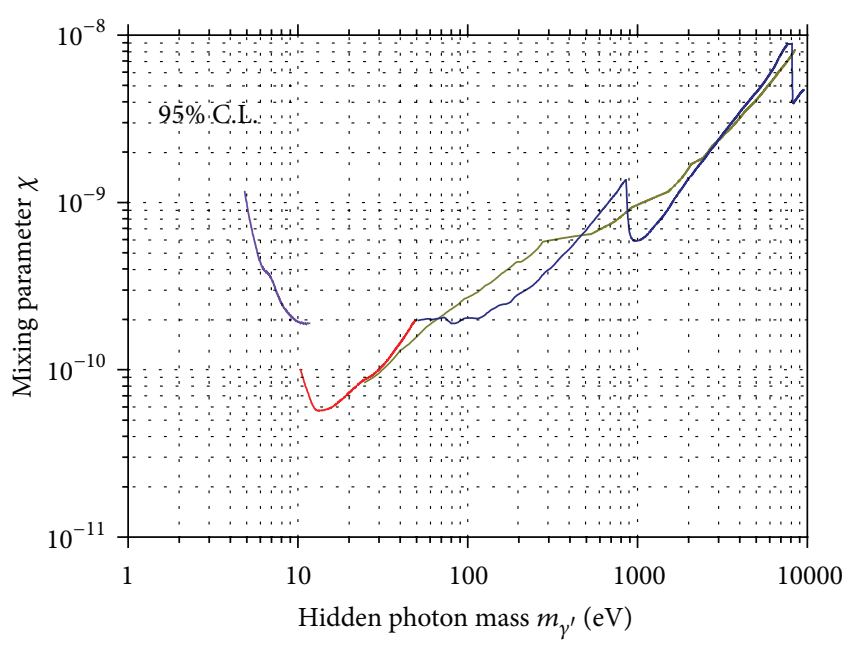

FIgURE 8: The limit for a mixing constant $\chi$.

equal to the energy needed to produce one ion pair in argon. This result has been obtained in direct measurements in a laboratory experiment by observing single electrons emitted from the surface of a copper cathode. At present time this is the only experiment using this technique which is based on measurement of the rate of single electron emission from a metal cathode of the counter sensitive only to HPphoton conversion and not sensitive to photon-HP conversion. Stellar astrophysics provides stringent constraints for this value. The limits obtained by using some astrophysical models (see, e.g., $[22,23]$ and references therein) are lower by several orders of magnitude. The limits obtained from stellar astrophysics are based on the models when both conversions are alike. The most impressive results have been obtained by observing electron emission from liquid xenon [23]. The threshold energy for the production of a single electron in liquid xenon is only $12 \mathrm{eV}$ and in a xenon detector the total fiducial volume is a target for CDM. To reach the comparable sensitivity we need to decrease the spurious count rate of single electron events of our detector by several orders of magnitude. We are not aware of any reasons why it cannot be realized in view that this is the first multicathode counter ever constructed. This question needs further study. We plan to make further improvements in the construction of MCC mainly with the aim of reducing the count rate of spurious pulses.

We consider that a key element in reducing the rate of spurious counts would be to use good isolating materials and very clean metals with meticulously polished surfaces and to assemble the counter in very clean dust-free environments. Approaching this strategy we hope to decrease the rate of spurious counts by about an order of magnitude. At this level, as our measurements with and without active and passive shields have shown, the background rate will be determined by ionized particles (muons and electrons) and by gammas from the material of passive shield. To decrease further the background rate we will go to underground laboratory, where the flux of muons is negligible in comparison with the one at a surface laboratory, and there we will also use a passive 


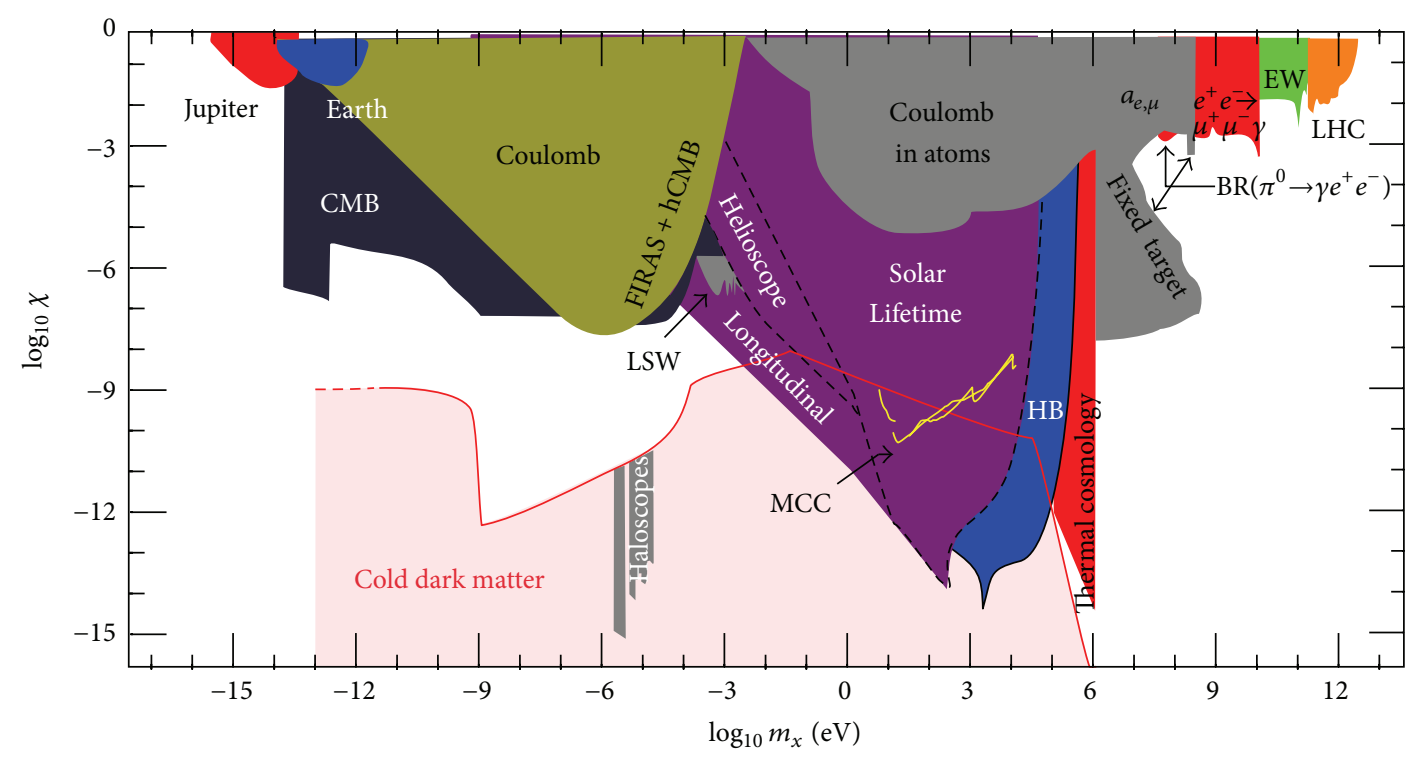

FIGURE 9: Constrained and still testable regions of photon/hidden photon mixing.

shield cabinet similar to the one we use now at the surface laboratory in Troitsk, Moscow region. Obviously the question of how far one can progress on this way can be solved only experimentally.

\section{Conclusion}

A new technique of multicathode counter (MCC) has been developed to search for hidden photon CDM in the assumption that all dark matter is composed of hidden photons (HPs). It was assumed also that if HPs have a mass greater than a work function of the metal of a cathode, they will induce emission of single electrons from a cathode. The technique used in this experiment is sensitive to HP-photon conversion and is not sensitive to photon-HP conversion. First preliminary result has been obtained for HPs with a mass from 5 to $500 \mathrm{eV}$ which demonstrates that this method works. The upper limit is above the ones obtained from stellar astrophysics by two to three orders of magnitude (see Figure 9 taken from [24]), but our result (MCC, yellow lines) has been obtained in direct measurements in a laboratory experiment. It is worthy also to note that our result has been obtained for the conversion of HPs of CDM into usual photon while the result obtained in astrophysics has been obtained for the conversion of photons in solar plasma in the depth of the sun into HPs, which is impossible to test experimentally.

Recently we are working with the aim of decreasing our limit substantially. Further progress will depend upon how successful our efforts will be to construct a detector with a lower count rate of spurious pulses. If they are successful and all sources of spurious pulses are eliminated, then the only source will be left, which can be eliminated by no means; it is a dark matter. The same will be true also for PMTs provided that dark matter is really composed of hidden photons and their mass is greater than a work function of a metal used for internal elements of detector. At present time we are constructing a new detector with a more developed design.

\section{Competing Interests}

The authors declare that there is no conflict of interests regarding the publication of this paper.

\section{Acknowledgments}

The authors express deep gratitude to E. P. Petrov and A. I. Egorov for very substantial contribution to fabrication of the counter and to Russian Government "Leading Scientific Schools of Russia” (Grant no. 3110.2014.2) for partial support in this work.

\section{References}

[1] K. A. Olive, K. Agashe, C. Amsler et al., "Review of particle physics," Chinese Physics C, vol. 38, no. 9, Article ID 090001, 2014.

[2] P. W. Graham, I. G. Irastorza, S. K. Lamoreaux, A. Lindner, and K. A. Van Bibber, "Experimental searches for the axion and axion-like particles," Annual Review of Nuclear and Particle Science, vol. 65, no. 1, pp. 485-514, 2015.

[3] L. B. Okun, "Limits on electrodynamics: paraphotons?" Soviet Physics-Journal of Experimental and Theoretical Physics, vol. 56, p. 502, 1982, Translated from Pis'ma v Zhurnal Experimental' noi i Teoreticheskoi Fiziki, vol. 83, p. 892, 1982.

[4] B. Holdom, "Two U(1)'s and $\epsilon$ charge shifts," Physics Letters B, vol. 166, no. 2, pp. 196-198, 1986.

[5] J. Suzuki, T. Horie, Y. Inoue, and M. Minowa, "Experimental search for hidden photon CDM in the $\mathrm{eV}$ mass range with a dish antenna," Journal of Cosmology and Astroparticle Physics, vol. 2015, no. 9, article 42, 2015.

[6] D. Horns, J. Jaeckel, A. Lindner, A. Lobanov, J. Redondo, and A. Ringwald, "Searching for WISPy cold dark matter with a dish 
antenna," Journal of Cosmology and Astroparticle Physics, vol. 4, article 16, 2013.

[7] D. H. Dowell and J. F. Schmerge, "The quantum efficiency and thermal emittance of metal photocathodes," Tech. Rep. SLACPUB-13535, 2009.

[8] A. V. Kopylov, I. V. Orekhov, and V. V. Petukhov, "Using a MultiCathode Counter (MCC) in the Search for Hidden Photon CDM," http://arxiv.org/abs/1509.03033.

[9] A. V. Kopylov, I. V. Orekhov, V. V. Petukhov, and A. E. Solomatin, "Gaseous detector with Sub-keV threshold to study neutrino scattering at low recoil energies," Advances in High Energy Physics, vol. 2014, Article ID 147046, 8 pages, 2014.

[10] A. V. Kopylov, I. V. Orekhov, V. V. Petukhov, and A. E. Solomatin, "Gaseous detector of ionizing radiation for registration of coherent neutrino scattering on nuclei," Technical Physics Letters, vol. 40, no. 3, pp. 185-187, 2014.

[11] M. Campbell, M. Chefdevilleb, P. Colas et al., "Detection of single electrons by means of a Micromegas-covered MediPix2 pixel CMOS readout circuit," Nuclear Instruments and Methods in Physics Research Section A: Accelerators, Spectrometers, Detectors and Associated Equipment, vol. 540, no. 2-3, pp. 295-304, 2005.

[12] H. S. Snyder, "Fluctuations for proportional counters," Physical Review, vol. 72, p. 181, 1947.

[13] G. D. Alkhazov, "Statistics of electron avalanches and ultimate resolution of proportional counters," Nuclear Instruments and Methods, vol. 89, pp. 155-165, 1970.

[14] A. F. Buzulutskov, "Gaseous photodetectors with solid photocathodes," Physics of Particles and Nuclei, vol. 39, no. 3, pp. 424453, 2008.

[15] Z. Ahmed, M. A. Bowles, R. Bunker et al., "A prototype low-background multiwire proportional chamber," Journal of Instrumentation, vol. 9, no. 1, Article ID P01009, 2014.

[16] G. F. Knoll, Radiation Detection and Measurement, John Wiley \& Sons, New York, NY, USA, 3rd edition, 2000.

[17] W. F. Krolikowski and W. E. Spicer, "Photoemission studies of the noble metals. I. Copper," Physical Review, vol. 185, no. 3, pp. 882-900, 1969.

[18] R. B. Cairns and J. A. Samson, "Metal photocathodes as secondary standards for absolute intensity measurements in the vacuum ultraviolet," Journal of the Optical Society of America, vol. 56, no. 11, pp. 1568-1573, 1966.

[19] R. H. Day, P. Lee, E. B. Saloman, and D. J. Nagel, "Photoelectric quantum efficiencies and filter window absorption coefficients from $20 \mathrm{eV}$ to $10 \mathrm{KeV}$,' Journal of Applied Physics, vol. 52, no. 11, pp. 6965-6973, 1981.

[20] H. Henneken, F. Scholze, M. Krumrey, and G. Ulm, "Quantum efficiencies of gold and copper photocathodes in the VUV and X-ray range," Metrologia, vol. 37, no. 5, pp. 485-488, 2000.

[21] E. Chevallay, J. Durand, S. Hutchins, G. Suberlucq, and M. Wurgel, "Photocathodes tested in the dc gun of the CERN photoemission laboratory," Nuclear Instruments and Methods in Physics Research Section A: Accelerators, Spectrometers, Detectors and Associated Equipment, vol. 340, no. 1, pp. 146-156, 1994.

[22] H. An, M. Pospelov, and J. Pradler, "Dark matter detectors as dark photon helioscopes," Physical Review Letters, vol. 111, no. 4, Article ID 041302, 2013.

[23] H. An, M. Pospelov, J. Pradler, and A. Ritz, "Direct detection constraints on dark photon dark matter," Physics Letters B, vol. 747, pp. 331-338, 2015.
[24] M. Klasen, M. Pohl, and G. Sigl, "Indirect and direct search for dark matter," Progress in Particle and Nuclear Physics, vol. 85, pp. $1-32,2015$. 

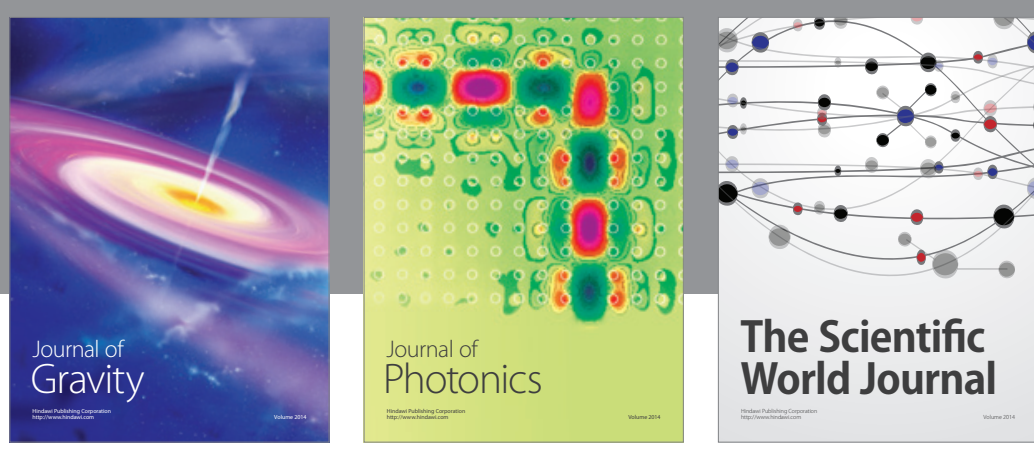

The Scientific World Journal
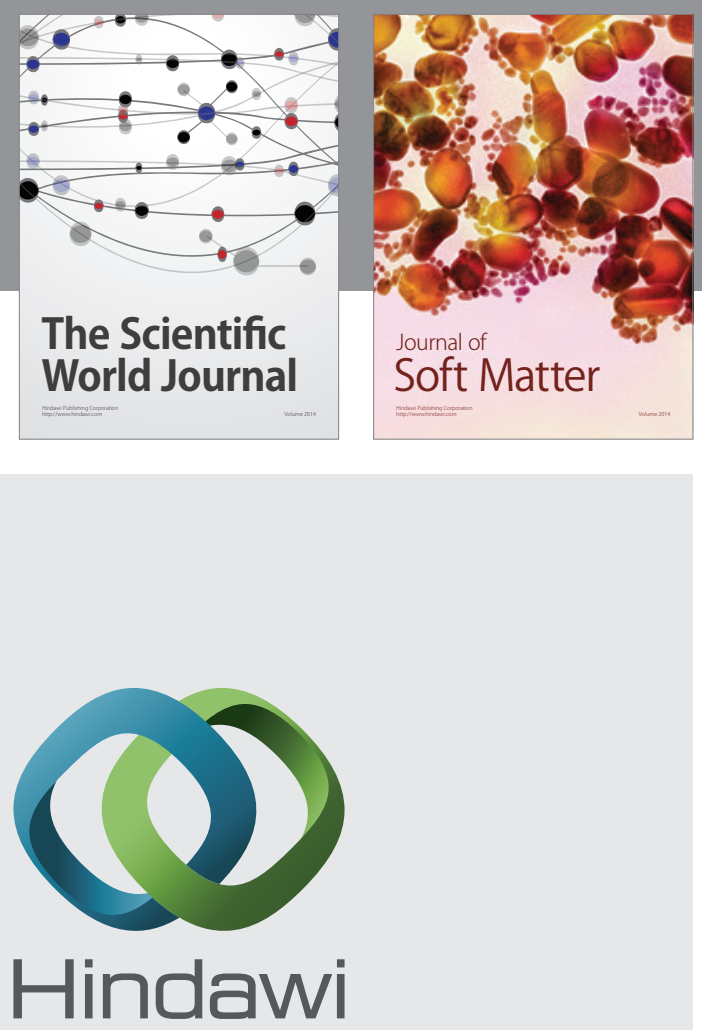

Submit your manuscripts at

http://www.hindawi.com

nternational Journal of

Statistical Mechanics
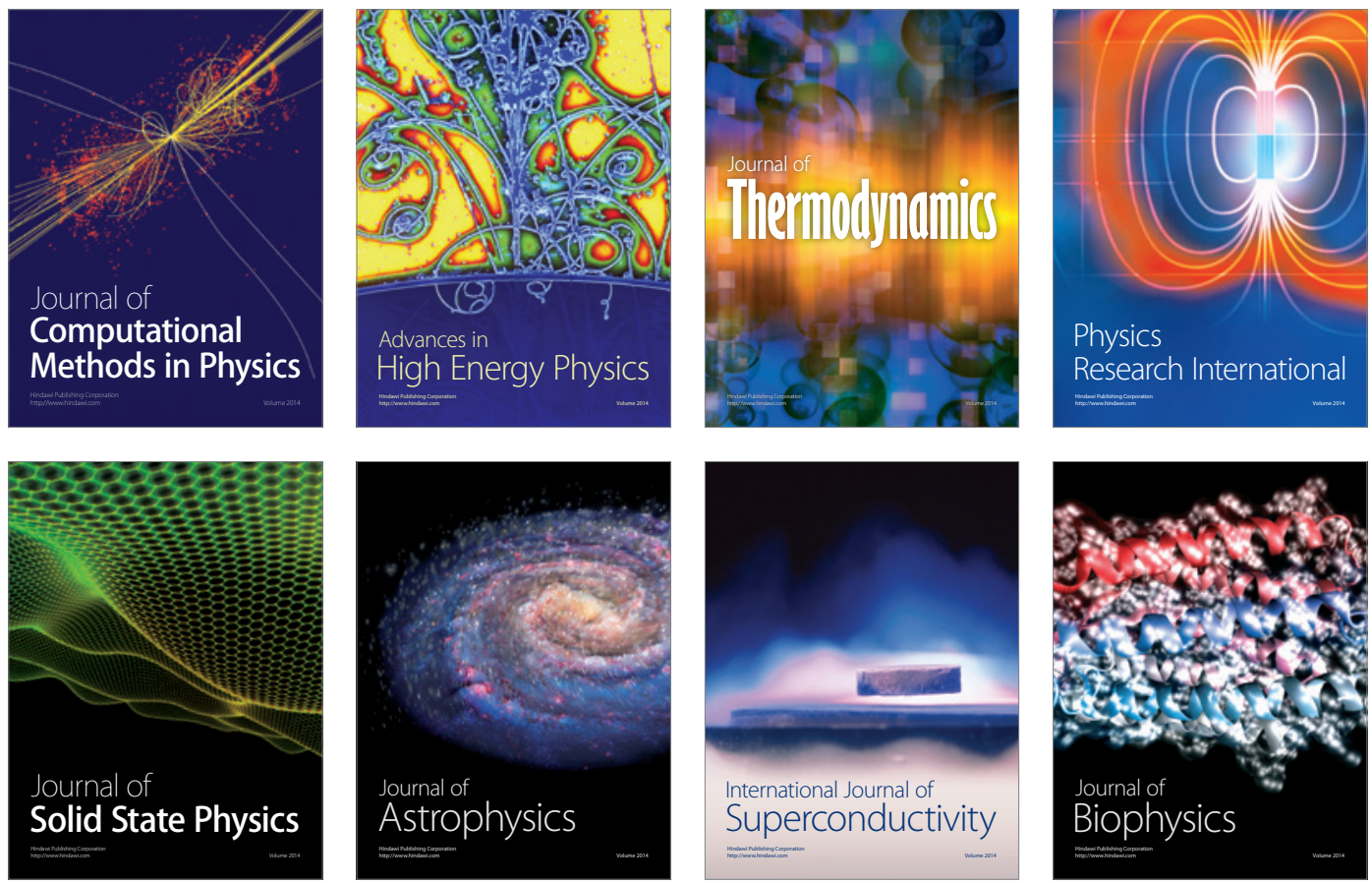
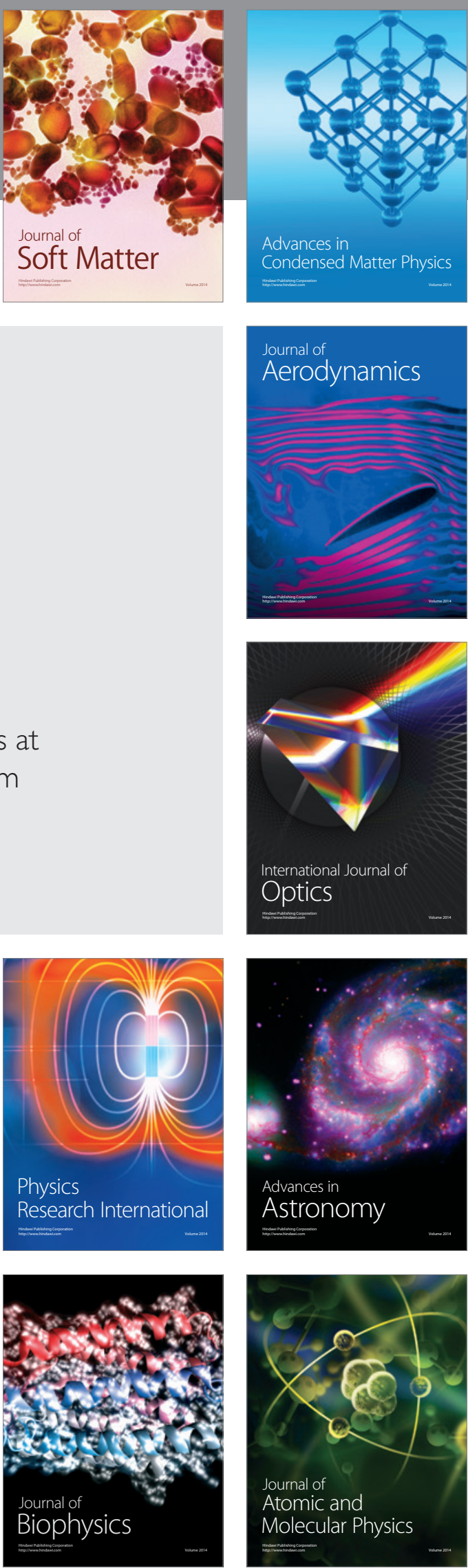